\title{
An Electromagnetic Forcing Device
}

\author{
An electromagnetic forcing mechanism is described which uses \\ feedback to reduce unwanted harmonics in the force \\ by M.W. Hyer, W.J. Anderson and R.A. Scott
}

\begin{abstract}
The paper describes the drawbacks of an electromagnetic forcing device, of the type commonly used to study forced vibration of structures, and presents details of a feedback control system designed to overcome them. The work described was initiated when attempting to generate sinusoidal forcing in a nonlinear beam-vibration study. Magneticmaterial nonlinearities and spatial inhomogenieties in the magnetic field led to unwanted harmonics in the force the beam experienced, and feedback was used to reduce these effects. A brief description of the principles of feedback control is presented and the problems encountered in applying the concepts to the electromagnetic forcing device are discussed. Details of the system, its problems, operating characteristics and limitations are presented.
\end{abstract}

\section{List of Symbols}

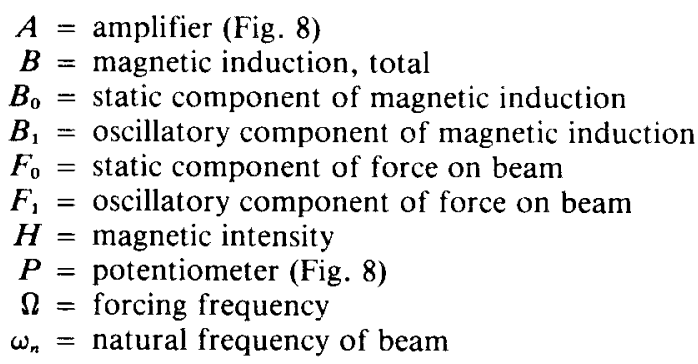

M.W. Hyer is associated with School of Engineering, Old Dominion University, Norfolk, VA 23508, W.J. Anderson and R.A. Scott are associated with Department of Aerospace Engineering and Department of Applied Mechanics, respectively, University of Michigan, Ann Arbor, MI 48109.

Paper was presented at 1977 SESA Spring Meeting held in Dallas, TX on May 15-20.

Work funded in part by the National Science Foundation, Grant No. $G K-36374 X$. It forms part of the PhD thesis of the first author at the University of Michigan in 1974.

\section{Electromagnetic Forcing}

There are many areas of engineering in which the response of a system to a pure-sinusoidal-force input is of considerable importance. However, generating a pure sinusoidal force can be quite difficult experimentally. One technique that has been tried in conjunction with ferromagnetic structures is the use of an electromagnetic coil driven by a harmonic signal generator and a power amplifier. Such a setup is shown schematically in Fig. 1. This method has the attractive features of no mechanical contact with the object being forced and being relatively simple to build. However, the method has several serious drawbacks. First, the structure actually feels a constant force plus a force alternating at twice the current frequency. Frequency doubling occurs because the ferromagnetic structure is attracted to a magnetic pole of either polarity and since the end of the magnet is a north pole once per cycle and a south pole once per cycle, the structure is pulled twice per cycle of alternating current in the coil. In addition, since the structure is always being attracted by the magnet, there is a nonzero mean attractive force. More formally, the force felt by the structure is pro-

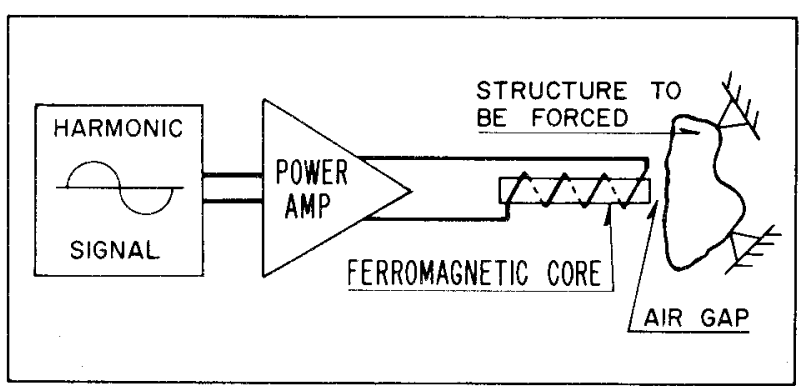

Fig. 1-Schematic of electromagnetic forcing device 


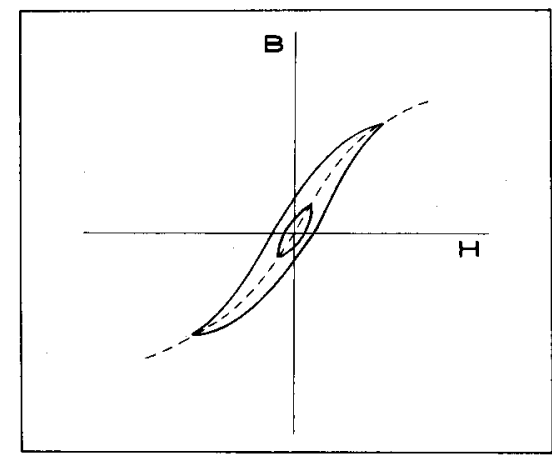

Fig. 2-Typical $B$ vs. $H$ relation

portional to the magnetic induction, $B$, squared. If the magnetic induction is $B_{0} \sin (\Omega t)$, then the force is proportional to $\left(B_{0}^{2} / 2\right)[1-\cos (2 \Omega t)]$. However, this is not strictly the case with the setup of Fig. 1. Since most ferromagnetic materials have a nonlinear relation between $B$ and $H$, the magnetic intensity, and since $H$ is directly proportional to the current in the windings, a harmonic current will not necessarily produce a harmonic $B$. Because of this, the force will contain harmonics of the current frequency. The character of the harmonics is determined by the current magnitude and the characteristics of the $B$ vs. $H$ relation for the material. A typical $B$ vs. $H$ relation, including hysteresis effects, is shown in Fig. 2. A second problem with this type of exciter is due to the strength of the magnetic field, and thus the force, being inversely proportional to the distance from the end of the coil. A qualitative indication of force variation with distance from the end of the coil is shown in Fig. 3. This means the structure is oscillating in a spatially nonuniform field. This, coupled with the temporal variation which may contain harmonics due to magnetic material nonlinearities, makes a force with a pure sinusoidal behavior difficult to achieve. When studying linear systems, it may be possible to account for the effects of these various harmonics in the response of the structure but, when studying the response of nonlinear systems, it would be difficult to determine what part of the harmonic content of the response is due to the system itself and what part is due to the deviation of the force from a pure harmonic. During the study of nonlinear beam dynamics, these problems led the authors to develop a feedback system for improving the quality of the force. The goal was to control the force on the beam as opposed, say, to its displacement.

\section{Feedback Control}

Figure 4 summarizes the elements inherent in the electromagnetic forcing device and Fig. 5 indicates the idea of simply closing the loop to force the output to follow the input. The system is a complicated electrical-magneticmechanical system and the control problem is not easy. Some of the problems were general problems of feedback control and some were associated with this device.

By far, the greatest problem with the system, as it is with many control systems, was stability. Instabilities in control systems arise from two main sources: slow time response of the system being controlled and unwanted noise in the system. As far as controllability is concerned, if a system is excited by a sinusoidal input, the ideal

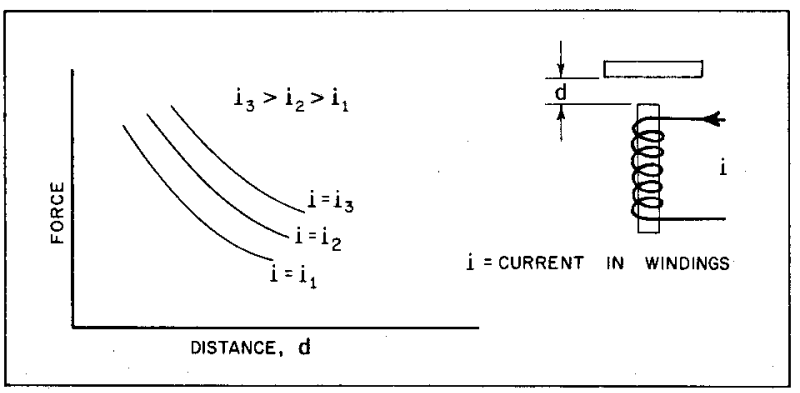

Fig. 3-Variation of force with distance from end of coil

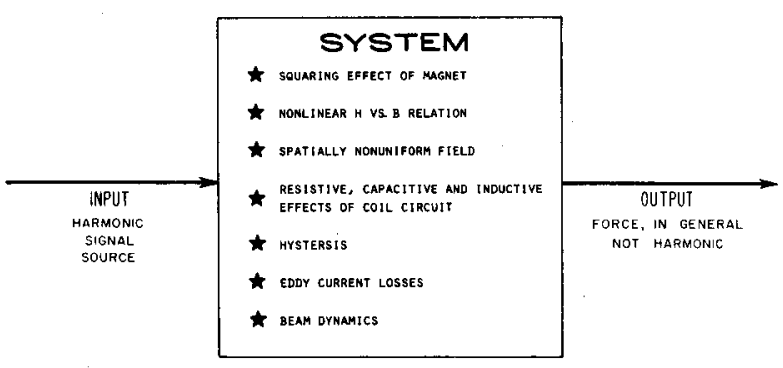

Fig. 4-Summary of the forcing system

situation would be to have the output be sinusoidal, exactly in phase with the input. Unfortunately, this is not the case in real systems and there is a frequencydependent phase difference between input and output which is detrimental to applying feedback. If, for example, the output is $180 \mathrm{deg}$ out of phase with the input, the subtraction of the output from the input at the summing junction is an addition and the actual input to the system becomes larger and the output increases without bound. In the forcing system, the prime sources of phase difference are the resistive and inductive effects of the coil circuit, and hysteresis and eddy-current losses in the magnetic materials.

When noise enters the system, say in the feedback loop between the output and the summing junction, it is interpreted as something the system being controlled is doing wrong. Part of the error signal, then, is a correction to compensate for this supposed wrongdoing of the system and the effects of the noise actually get into the output. If the noise is of the right frequency, its phase on output can be shifted such that it adds to the original noise source and the system becomes unstable. In the system at hand, the main sources of noise were $60-\mathrm{Hz}$ ripple and random noise in the circuitry of the various transducers, filters and amplifiers.

An additional problem when applying feedback control to a system is the loss of output amplitude. Subtracting some or all of the output from the original input reduces the actual input to the system and so reduces the output. This can be remedied by amplifying the error signal, or as it is commonly called, increasing the gain. However, gain cannot be increased at will because the noise and phase effects will be amplified as well and, eventually, the system loses stability.

Two problems peculiar to applying feedback to the electromagnetic forcing system were the problem of frequency doubling and the problem of measuring the force accurately. Because of frequency doubling, at the 


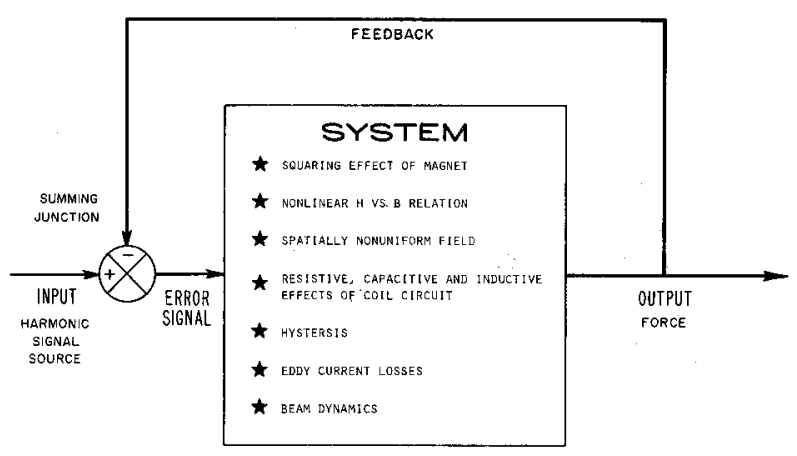

Fig. 5-Application of feedback to the forcing system

summing junction a signal of frequency $\Omega$ was being compared with a signal of fundamental frequency $2 \Omega$. Such a comparison was meaningless and this serious problem had to be overcome to even begin. The problem of measuring the force the structure felt was important, since it was the force that was being controlled and it had to be represented as accurately as possible.

Successful designs of such systems can be achieved, as witnessed by the work of Plas and Janssen' in the design of machine tools. They controlled magnetic induction rather than force but it is not clear from their work how the forcing frequency compared with the resonant frequencies of the cutting tool and the object being machined. For the application at hand, this is an important point, since the large displacements associated with resonant frequencies of the beam being forced caused greater distortions in the force than at other frequencies. The design of the forcing system presented here is the result of several iterations on a system which had been previously used for beam-vibration studies but did not incorporate feedback. Each successive iteration was designed to overcome some of the aforementioned problems in applying feedback. The design is not completely satisfactory and, to aid future investigators, several alternative procedures that were tried are mentioned briefly. A more complete account of the evolution of the design can be found in Ref. 2.

\section{Design of the Unit}

To put the problem in context, the complete experimental setup will be briefly described. The beams being studied were clamped-clamped, three-layer beams with thin elastic outer layers and viscoelastic cores. ${ }^{3}$ The beams were $11 \mathrm{in.}(279 \mathrm{~mm})$ long, $1 \mathrm{in}$. $(25.4 \mathrm{~mm})$ wide and of various thicknesses, in the range $.066 \mathrm{in}$. $(1.67 \mathrm{~mm})$ to $.1 \mathrm{in} .(2.54 \mathrm{~mm})$. They were mounted in a fixture built around a lathe bed and were forced at midspan. Referring to Fig. 6, a lathe bed had been chosen because the traveling tool bed could be used as a convenient transducer mount for measuring the response of the beam specimen along its entire length. Within the end pieces, which were bolted to the lathe bed, was a set of steel jaws which actually provided the clamping mechanism. The beam nonlinearity arose due to axial stretching. Two 1-in. $(25.4-\mathrm{mm})$ by 3 -in. $(76.2-\mathrm{mm})$ steel bars were bolted between the tops of the end pieces to give the support fixture additional rigidity. The main concerns were the amplitude of the beam displacement as a function of forcing frequency and the frequency content of the displacement response.

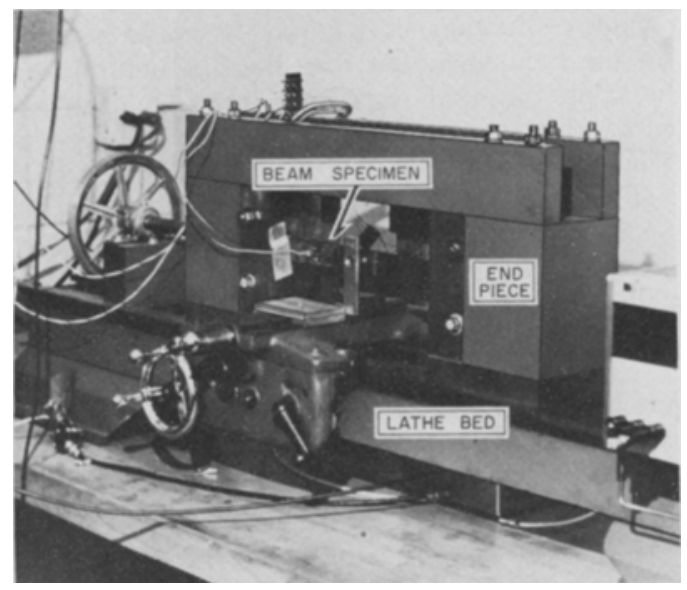

Fig. 6-Experimental setup, overall front view

To measure the force on the beam, two schemes were tried, a free magnet and a fixed magnet. If the magnet were floating free in space, then by Newton's Third Law, the force on it would be equal and opposite the force on the beam. If the magnet were a known point mass and if the acceleration were known, then the force on it and the beam would be known. This situation can be closely approximated by suspending a magnet on fine wires, such that the natural pendulum frequency is much lower than the forcing frequency, and measuring its acceleration with an accelerometer. This scheme was tried but, from the point of view of feedback, the results were not satisfactory. The magnet and accelerometer had a mass of $.00285 \mathrm{lb}-\mathrm{s}^{2} / \mathrm{in} .(.499 \mathrm{~kg})$. The accelerometer was a piezoelectric device and, with forces on the beam in the range of .1 lb $(.448 \mathrm{~N})$, it was operating at a low acceleration level. The resulting output signal-to-noise ratio of the accelerometer was low. In addition, in the range $25-50 \mathrm{~Hz}$, which was within the range of interest, the frequency response of the accelerometer was not flat. Since excessive noise and poor frequency response cannot be tolerated in a feedback system and, since no satisfactory accelerometer could be obtained, the free suspension system was abandoned. Piezoresistive accelerometers existed which were good for low acceleration levels but their natural frequencies were too close to the forcing frequencies used in the experiment.

The fixed magnet approach was then adopted with some degree of success. In principle, the force on the beam could have been determined by mounting the magnet rigidly and measuring the force on the mount. Since nothing is actually rigid, this is tantamount to keeping the natural frequencies of the magnet-mount system high compared to the forcing frequencies so inertial effects of the magnet do not substantially affect the force measurement. In order to keep the signal-to-noise ratio as high as possible, a variation on the approach was employed. The mount was made somewhat flexible and displacements were used as a measure of force. Referring to Fig. 7, a vertical strut was attached to the midpoint of a 1 -in. (25.4-mm) square horizontal steel bar which, in turn, was connected to the steel end pieces of the support fixture. A magnet was mounted on the strut 1.5 in. $(38.1 \mathrm{~mm})$ from the horizontal bar. The strut was extended upward another 2.5 in. $(63.7 \mathrm{~mm})$, the plan being to use the strut-tip deflection as a measure of the force the magnet exerted on the beam. This extension gave a displacement larger than one which would have been obtained if measurements 
were made at the magnet location. It should be noted that for forcing frequencies less than the first natural frequency of the strut, the strut response could be closely approximated by that of a single-degree-of-freedom system and, for such a system, the dynamic correction factor is 1 percent at .1 of the natural frequency and 4 percent at .2 of the natural frequency. Thus, for a fixed force level, the displacement of the strut remained essentially constant over this frequency range and with a static calibration, using known weights, could be used to measure force.

The strut was made of a nonferromagnetic material, aluminum (for a reason to be described later), and its lowest natural frequency was $466 \mathrm{~Hz}$. The magnet was 250 turns of No. 26 enameled copper wire on a .375-in. (9.52-mm) diam core. Choosing the dimensions of the strut was a trade-off between keeping the natural frequency high and, at the same time, having the tip displacement large enough to give a high signal-to-noise ratio for the displacement measuring device, a Bentley-Nevada Model 316 proximeter. The proximeter is nonlinear over its entire operating range but, in this application, the displacements of the strut tip were so small that the nonlinearity of the proximeter response could be ignored. The output of the proximeter, then, was a measure of the force on the beam and was fed back to be compared with the reference sinusoid.

As mentioned previously, because of the squaring effect of the magnet, the forcing frequency was twice the frequency of the oscillator. Several steps were taken to overcome the problem. One step was to superimpose the alternating magnetic field on a static field generated by direct current through the coil. In this case, the magnetic induction was

$$
B=B_{0}+B_{1} \sin (\Omega t)
$$

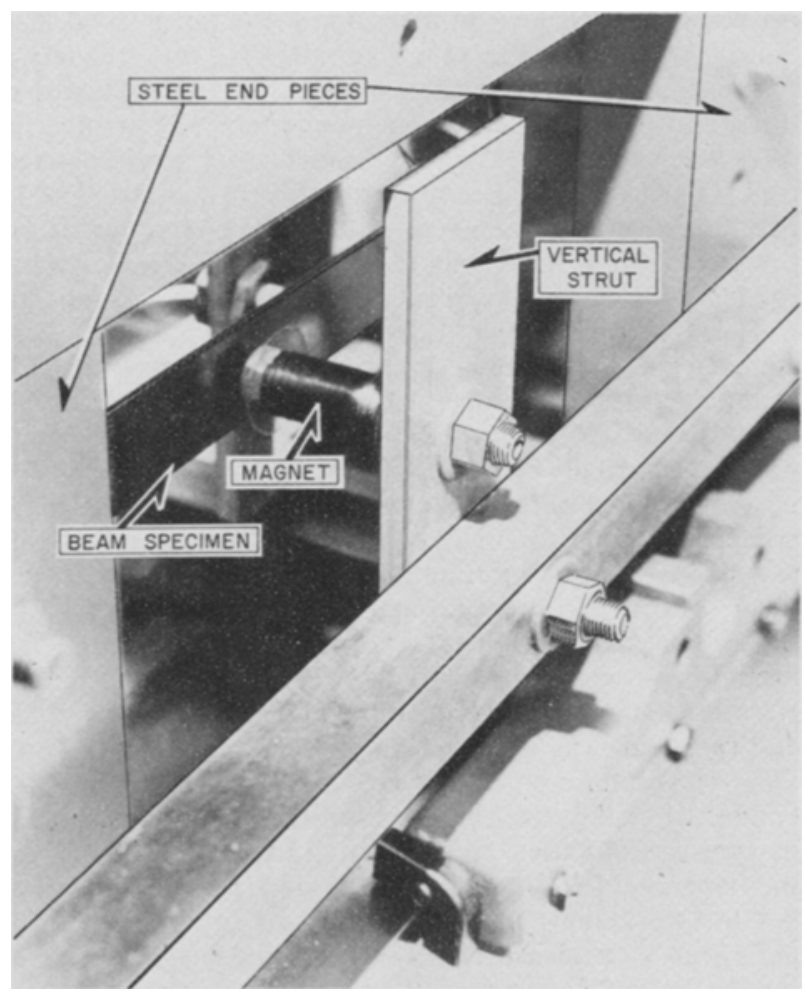

Fig. 7-Fixed magnet, rear view
Then the force was proportional to $B^{2}$, i.e.,

$B_{0}^{2}\left\{\left[1+1 / 2\left(B_{1} / B_{0}\right)^{2}\right]+2 B_{1} / B_{0} \sin \Omega t-1 / 4\left(B_{1} / B_{0}\right) \cos (2 \Omega t)\right\}$

Hence, for $B_{0} \geq B_{1}$, the $2-\Omega$ component in the force was considerably weakened. For example, if $B_{0}=B_{1}$, there is about a 25 -percent component of $B$ at $2 \Omega$ and, if $B_{0}=$ $4 B_{1}$, the $2-\Omega$ component is about 6 percent. Of course, the force felt by the beam was a static force plus an oscillatory component.

Another step to counter the squaring effect of the magnet was to build a square-rooting device into the circuit. For $B_{0} \geq B_{1}$, the voltages and currents associated with $B$ were always positive, or zero, and it was possible to build a circuit which took the square root of the voltage driving the coil.

Figure 8 shows the final configuration of the feedback system. The backbone of the system was a ten-amplifier analog computer. The voltage signal from the reference oscillator and the voltage signal from the force proximeter were compared at the summing junction, basically a summing amplifier. Amplifier 5 inverted the feedback so that amplifier 10 actually subtracted the two signals. A static voltage to produce the static magnetic field was added to the alternating field at amplifier 7. This was accomplished by stepping down a $100-\mathrm{V}$ source. Amplifiers 6 and 9 and the 37-segment diode multiplier made up the square-rooting circuitry. The idea was to approximate the nonlinear square-root relation by a piece-wise linear relation using a series of diodes and variable resistors. The 37 segments provided good resolution to the square-root approximation. The magnet itself was driven by a power amplifier built using an RCA HC-2500 solid-state operational amplifier, a high output-current device. Shown also in Fig. 8 is an inner feedback loop around the power amplifier. This loop went from the ground resistor of the coil circuit to amplifier 8 . The function of this loop was to reduce the phase difference between the voltage into the power amplifier and the current one, making the amplifier more like a voltage-controlled current source. For low values of force, say $.01 \mathrm{lb}(.0448 \mathrm{~N})$, the phase distortion could be reduced to 1 deg over the frequency range of interest. However, for larger values of force, it was not possible to feed back the same amount of voltage from the coil and use the same gain without causing an instability in the inner loop. Thus the $1 \mathrm{deg}$ could not be realized at all force levels.

In the feedback loop, a capacitor was necessary to block the constant voltage due to the static air gap associated with the stand-off distance of the proximeter probe. The constant voltage was in the range $6-10 \mathrm{~V}$ and would have saturated the d-c amplifier which was designed to amplify voltage in the milli-volt range. The d-c-coupled, low-pass filter was in the feedback loop to attenuate the strut resonant frequency and other unwanted high-frequency noise. Within the system, amplifier gains and potentiometer settings were adjusted so as to use the full operating voltage ranges of the various components.

\section{Characteristics of the System}

The system shown in Fig. 8 produced a reasonably clean harmonic force. An on-line spectrum analyzer was used to monitor the harmonic content of the force and to determine the effects of feedback. Figure 9 shows the spectrum of the force signal, with and without feedback, when the reference signal from the oscillator was $30 \mathrm{~Hz}$. Feedback reduced the intensity level of the $2-\Omega$ component 
Fig. 8-Configuration of feedback system

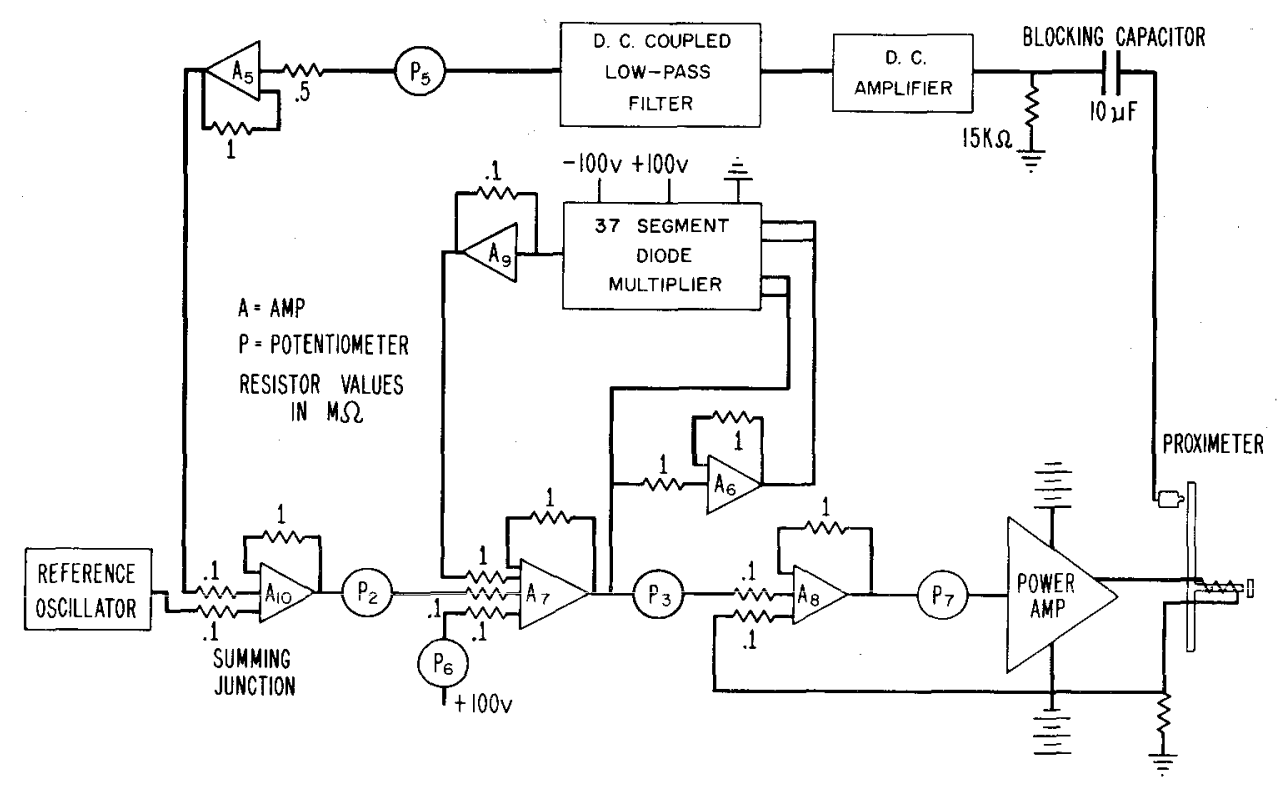

by about $8 \mathrm{db}$. In terms of amplitude, this means the amplitude of the first harmonic was reduced from 7.8 percent of the fundamental to 3.1 percent. The peak in the vicinity of $466 \mathrm{~Hz}$ stemmed from the strut's lowest natural frequency. A limiting feature of this design was the broadband noise centered at $275 \mathrm{~Hz}$. Unfortunately, this noise led to an instability when the gain was increased above a certain level. Its source was finally traced to the phase distortion of the low-pass filter, an important component of the system since its removal allowed the strut resonant frequency to enter the system and caused an instability at $466 \mathrm{~Hz}$.

When evaluating the feedback system, it must be recalled that the system includes the beam being forced and that the output, the force on the beam, was a strong function of the components in the system, in particular, the beam dynamics. This system was designed to force highly damped beams with resonant frequencies of $50-60 \mathrm{~Hz}$. The system was tuned to meet the demands of these beams and if beams of higher or lower frequency or more or less damping were to be examined, the system components more than likely would have to be redesigned. In addition, the system was nonlinear, both due to the beam and to the magnetic effects and, thus, its characteristics were amplitude dependent.

Figure 10 shows additional characteristics of the system for the case of the static force, $F_{0}$, equal to $.15 \mathrm{lb}(.667$ $\mathrm{N})$ and the oscillatory force, $F_{1}$, equal to $.05 \mathrm{lb}(.222 \mathrm{~N})$, typical values for the experiment. As can be seen, feedback reduced the percentage of the $2-\Omega$ component in the force over all frequencies but, as the beam approached resonance, the large displacements of the beam caused an increasing amount of the 2- $\Omega$ component. Shown also in this figure are the amounts of phase shift caused by various components of the system. These phase distortions are the key element in this and any feedback system. Ideally, when feedback is employed, there should be no phase difference between the input and output because the output is exactly following the input. The phase characteristics in an actual system, before feedback is applied, indicate the degree of difficulty in successfully applying feedback. In Fig. 10, the phase distortion between input and output with no feedback was measured by comparing input and output signals at the summing junction without actually connecting the feedback. Thus, it represents the cumulative effects of all elements in the forward and feedback circuits. The phase effects of the individual components were measured by isolating them from the circuit. The $d-c$ amplifier contributed a negligible amount but the low-pass filter, a four-pole Butterworth, produced a significant amount as did the resistive-inductive effects of the coil circuit. Hysteresis and eddy-current dissipation in the magnetic materials accounted for the rest of the total phase difference. From the figures, it is evident feedback had an effect on reducing phase but, near beam resonance, the phase distortion was still significant. As mentioned previously, an instability of $275 \mathrm{~Hz}$, due to broadband noise, limited the effectiveness of this design.

It might be noted that phase differences between the input and output of a component, such as a filter, were often the reason for the unsuitability of that component. That was the reason for rejecting a scheme that could have led to a major improvement in the level of force.

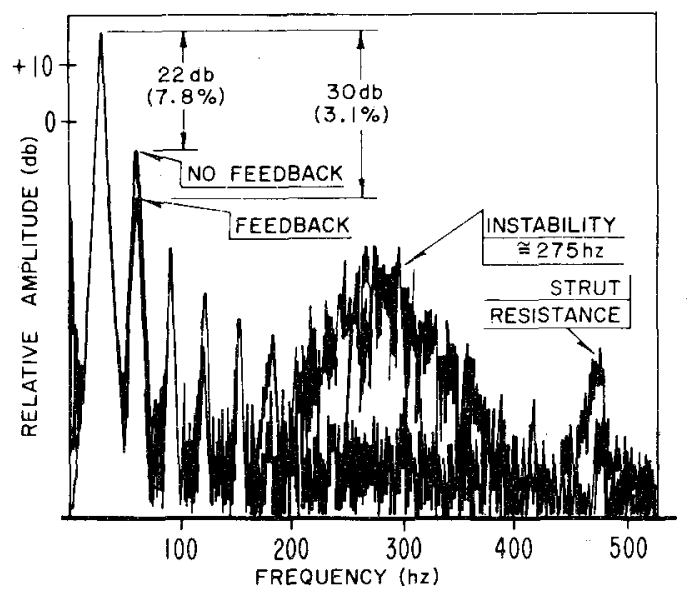

Fig. 9-30-Hz forcing spectrum, with and without feedback 
Fig. 10-Characteristics of the system

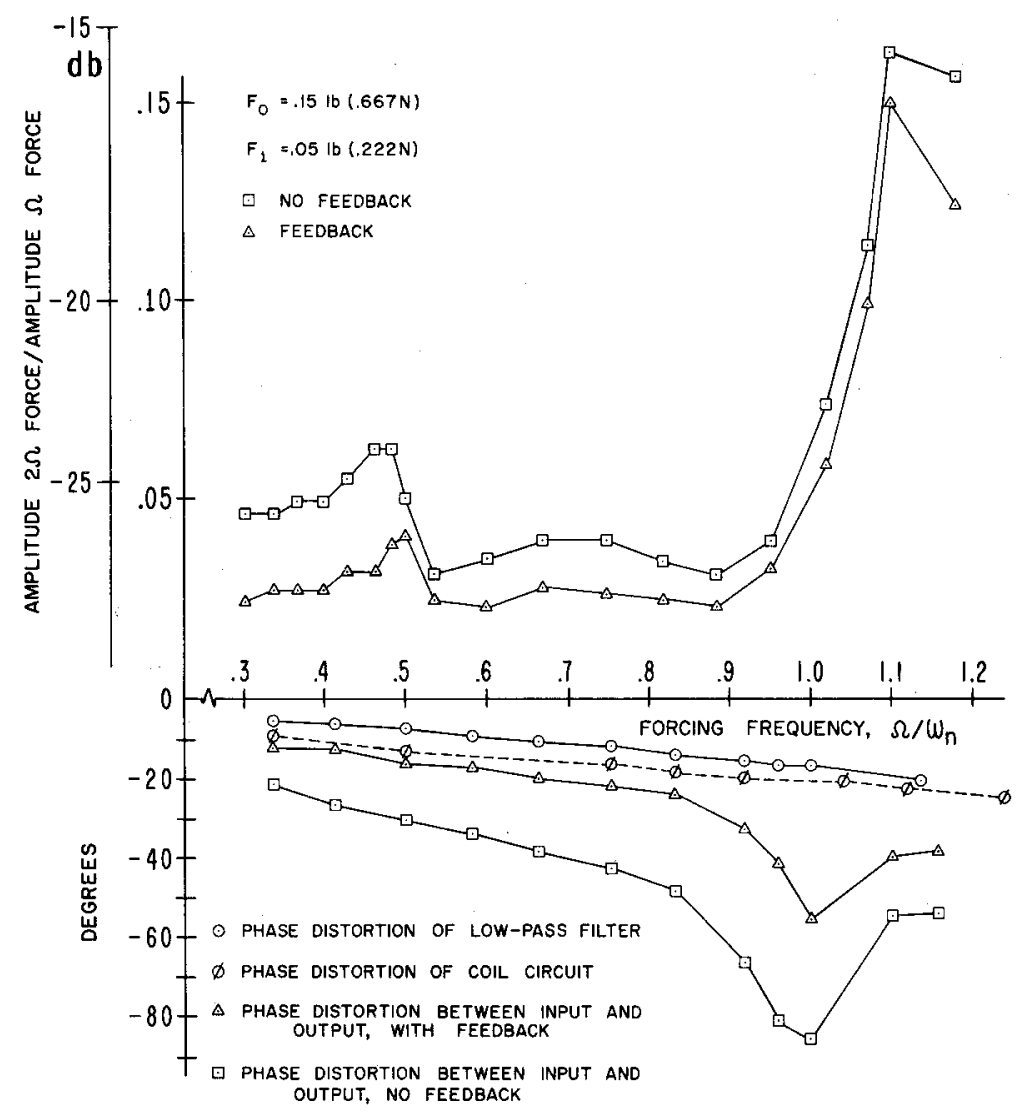

This idea hinged around minimizing the fringing effect of the lines of force coming from the magnet. If the strut were made of a ferromagnetic material such as steel, then the flux lines would have a good magnetic path from the magnet, through the beam specimen, into the end pieces and back through the horizontal bar and strut to the magnet. In this way higher force levels could have been achieved since almost all of the flux lines passed through the beam. However, doing this created a magnetic circuit whose reluctance varied periodically according to beam dynamics because the air gap, which was the major source of reluctance, depended on beam displacement. It was impossible to apply feedback to such a system because the force on the beam was substantially out of phase with the reference oscillator and, when feedback was applied, the system went unstable. Consequentially, it was decided to break the magnetic circuit by making the strut out of aluminum.

In conclusion, it can be said that the system presented in Fig. 8 was moderately successful in improving the quality of force and represents a first step in overcoming the serious drawbacks of electromagnetic forcing. Overcoming these drawbacks is important because an accurate noncontacting forcing mechanism would complement the various noncontacting displacement-measuring instruments available. The work presented here provides a reference and direction for future efforts.

\section{Recommendations}

There are several areas for improvements on the design. As Figs. 9 and 10 show, the amount of $2-\Omega$ component and the amount of phase distortion still depend strongly on the response of the beam specimen. This indicates that the specimen still affects the overall magnetic-field strength. Constructing the horizontal bar from aluminum and using a brass bolt and nut to hold the strut to the bar had no effect on reducing the beam-response dependency. So, it is conjectured that the steel lathe bed, the steel end pieces, the steel support pieces across the top and the steel in the beam specimen are coupled, magnetically, to a significant degree. This would suggest that, in designing a new installation, all ferromagnetic materials should be eliminated from the support fixture. Another improvement would be to replace the low-pass filter with a narrow band-reject filter tuned to reject the strut natural frequency. Such a device would have to be made to have more favorable phase-distortion characteristics. The nonlinear relation between $B$ and $H$ could be countered by using another inner feedback loop to control the relation between $B$ and the input to the power amplifier. Such a loop would also help minimize phase differences in that part of the system which were due to hysteresis and eddy currents. $B$ could be measured with a small search coil or a Halleffect probe.

\section{References}

1. Plas, J. and Janssen, L., "A Transistorized Contactless Electromagnetic Exciter," Int. J. Machine Tool Design Research, 12, 1-16 (Mar. 1972).

2. Hyer, M.W., "Nonlinear Vibration of Three-Layer Beams," PhD Thesis, University of Michigan, University Microfilm, Ann Arbor, MI (1974).

3. Hyer, M.W., Anderson, W.J. and Scott, R.A., "Nonlinear Vibrations of Three-Layer Beams with Viscoelastic Cores: I, Theory," J. Sound and Vibration, 46 (1), 121-136 (1978). 